\title{
An Ultrasonic Filterbank with Spiking Neurons
}

\author{
Hisham Abdalla \\ Electrical and Computer Engineering Dept \\ Institute for Systems Research \\ University of Maryland \\ College Park, Maryland, U.S.A. \\ hisham@isr.umd.edu
}

\author{
Timothy K. Horiuchi \\ Electrical and Computer Engineering Dept \\ Institute for Systems Research \\ Neuroscience and Cognitive Science Program \\ University of Maryland \\ College Park, Maryland, U.S.A.
}

\begin{abstract}
To support our ongoing work in modeling bat echolocation, a binaural, ultrasonic cochlea-like filter bank has been designed with moderate quality $(Q)$ factor (as high as 65 ) with spiking neurons that are driven by the filter outputs. The neuron addresses are reported off chip at the time of the spike in an unarbitrated fashion and in current-mode to reduce the amount of capacitively-coupled feedback into the filters. This chip was fabricated in a commercially-available $0.5 \mu \mathrm{m}$ CMOS process and consumes 0.425 milliwatts at 5 volts.
\end{abstract}

\section{INTRODUCTION}

Echolocating bats specialize in high-frequency hearing using echolocation sounds that typically range in frequency from $20 \mathrm{kHz}$ to $100 \mathrm{kHz}$ [1]. While some bats are specialized for specific frequencies with cochlear filtering at extremely high threshold $\mathrm{Q}_{10 \mathrm{~dB}}{ }^{1}$ (e.g., 400) we are studying bats that use a broadband vocalization and are ultrasonic frequency generalists (e.g., Myotis lucifugus) with $\mathrm{Q}_{10 \mathrm{~dB}}$ values in the range of 10 to 30 [1]. Good frequency resolution is important for vertical localization, discriminating close objects, as well as for prey discrimination.

The development of silicon models of the mammalian cochlea for audio frequencies has been dominated by the cascaded filter design proposed by Lyon and Mead [2]. This model provides a good fit to the biological cochlea tuning curve shape, phase response, and logarithmic frequency representation [3]. This model is also space and power efficient in its implementation, utilizing preceding filters to dramatically increase the sharpness and phase roll-off on the high-frequency side of the tuning curve. While many significant improvements to this design have been made (e.g., a 2-D fluid model [4, 5], current domain signals [5], local gain control for managing oscillations [6], and non-volatile on-chip corrections), obtaining high $\mathrm{Q}$ values and having many stages in a small frequency range remain a challenge.

\footnotetext{
${ }^{1} \mathrm{Q}_{10 \mathrm{~dB}}$ values, a standard biological measure of the response threshold tuning sharpness, are not directly comparable to conventional Q-values measured from bandpass filters because the measure different bandwidths.
}

The parallel filterbank approach, most common in software approaches, involves the space-consuming construction of high-order bandpass filters to replicate biological tuning curves, making them somewhat impractical to implement in hardware. Furth and Andreou [8], however, proposed a hybrid construction which uses a non-resonant lowpass filter cascade which drives low-order bandpass filters from each tap in the cascade, using a gyrator-based bandpass filter [9]. Integrating resonant filters and high-speed digital neurons has proven to be a technical challenge and most examples of spike-based auditory feature extraction have used multiple chips (e.g. [10]).

In this paper we describe a binaural ultrasonic filterbank using the gyrator-based filter that achieves moderate Q-value bandpass filtering followed by spiking neurons for use in modeling the bat echolocation system. The filter frequencies are spaced exponentially to mimic the frequency spacing in the bat cochlea. In addition to filtering, we designed a neuron circuit that produces minimal spike-related power-supply noise and reports spike-events using a current-mode digital output.

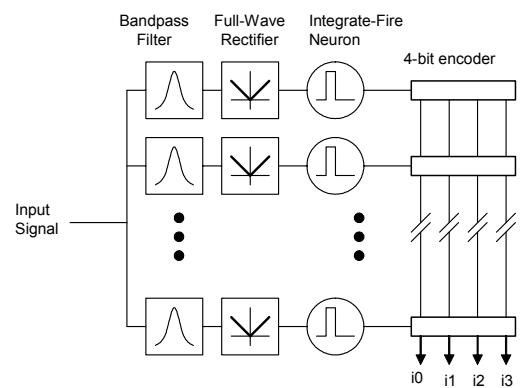

Fig. 1. Block Diagram of one cochlea-like ultrasonic filter bank. The input signal is provided in parallel to all filters. The gyratorbased bandpass filters are created with exponentially-increasing center frequencies. The outputs are then full-wave rectified and converted to currents that are injected into an integrate-and-fire neuron. The output spikes generate fixed-value inward or outward current pulses on the address lines to represent the address.

\section{System AND CiRCUIT DESIGN}

The overall system architecture for one filterbank is shown in Figure 1. The chip contains two identical, independent filter banks, thus we only discuss one in the sections to follow.

This work was supported in part by the Air Force Office of Scientific Research (FA95500410130) and in part by the National Science Foundation under (CCF0347573) 


\section{A. Gyrator-based Bandpass Filter}

The bandpass filter is based on the simple RLC-equivalent circuit shown in Figure 2. This architecture, implemented earlier by Furth [9], is a tunable high-Q bandpass filter. We implemented this architecture using source-degenerated, differential-pair transconductance amplifiers (transamp)(see Figure 3) operated in the transistor's subthreshold region of operation. The center frequency of each filter is set using a resistive ladder which produces a linear sequence of bias voltages across the array. Because the bias current for $g_{m 2}$ is exponentially related to the bias voltage, the filter frequencies vary exponentially across the array.

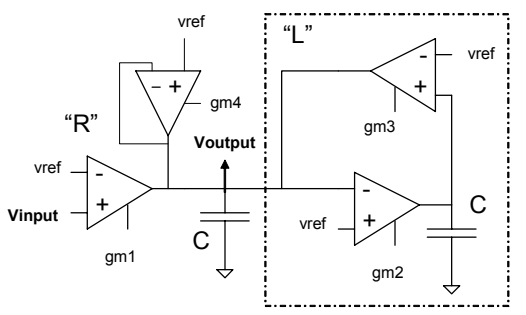

Fig. 2. The gyrator-based bandpass filter. Two transconductance amplifiers are used to generate the resistance $\mathrm{R}$ to ensure that large input signals will not trigger oscillations due to the saturating nature of the amplifiers. The two amplifiers that function as the inductance $\mathrm{L}$, are shown inside the dotted box.

The transfer function of this filter is given by:

$$
|H(s)|=\frac{K s}{s^{2}+s\left(\frac{\omega_{o}}{Q}\right)+\omega_{o}^{2}}
$$

where $\omega_{o}=\frac{\sqrt{g_{m 2} g_{m 3}}}{C}$ and $Q=\frac{\sqrt{g_{m 2} g_{m 3}}}{g_{m 4}} \quad K=\frac{g_{m 1}}{C}$.

The center frequency $\omega_{c}$ as derived from the pole-frequency analysis is given by:

$$
\omega_{c}=\omega_{o} \sqrt{1-\frac{1}{4 Q^{2}}}=\frac{1}{2 C} \sqrt{4 g_{m 2} g_{m 3}-g_{m 4}^{2}}
$$

which approximately equals $\omega_{0}$ for large values of $Q$. The transconductance of the $i^{\text {th }}$ amplifier $\left(\mathrm{g}_{\mathrm{mi}}\right)$ is given by:

$$
g_{m i}=\frac{\kappa^{2}}{(1+\kappa) 2 V_{T}} I_{b i}
$$

where $\kappa$ is the body effect, $V_{T}$ is the thermal voltage, $\mathrm{I}_{\mathrm{bi}}$ is the bias current of the $i^{\text {th }}$ amplifier.

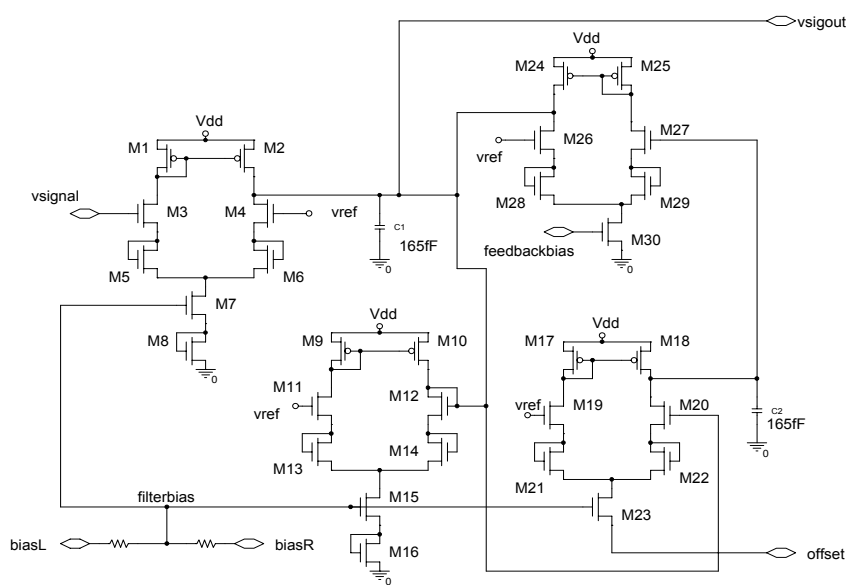

Fig. 3. Transistor implementation of the gyrator-based circuit. The bias voltage filterbias is specified by the linearly spaced voltages on a resistor ladder. The bias currents in M7 and M15 are related to the current in M23 by a power of approximately 0.41 to reduce the dependence of $\mathrm{Q}$ on the center frequency. feedbackbias and offset are the same for all filters. (W/L values in microns: $\mathrm{M} 15=3.9 / 3.0 ; \mathrm{M} 30=$ 3.6/3.6; All other transistor $\mathrm{s}=3.6 / 3.0$ )

\section{B. Temporal Derivative and Rectification}

Figure 4 shows the circuit that transforms the filter output amplitude (vsigout) into a current signal (into the node labeled vmem) that will drive the neuron circuits to their spiking threshold. The gain of the output current mirror is controlled with the voltage syntilt. One consequence of the temporal derivative is that neurons for higher frequencies receive more current for the same amplitude signal than at lower frequencies.

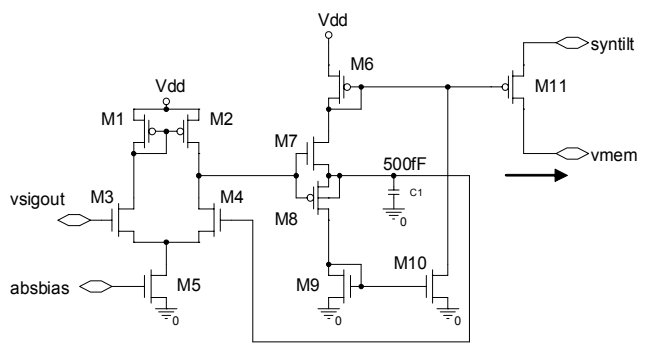

Fig. 4. Full-wave rectifier circuit. This circuit produces an attenuated copy of the current required to charge the capacitor $\mathrm{C} 1$ to follow the input signal. This is functionally a temporal derivative. The signal is rectified and the current is sent to the neuron. This output current can be reduced in the output current mirror by reducing the voltage syntilt down from Vdd. (W/L values in microns: M1, M2, M3, M4 = 1.8/3.6; M5 = 3.6/3.6; M6, M9, M10 = 1.8/1.8; M7, M8 = 1.2/1.2; M11 = $6.3 / 1.5)$

\section{A Low Supply-Noise Neuron}

The neuron circuit was designed to integrate charge and produce a pulse event once a voltage threshold was crossed. The differential pair structure maintains a constant current draw on Vdd even during the spike, drawing the positive feedback current from the digital Vdd line ( $\mathrm{dVdd}$ ). The positive feedback current is a pulse that is equal to 1.63 times the bias current in M3. This current pulse is used to: 1) generate the spike, 2) reset the membrane potential via the 
refractory period mechanism, and 3) produce the address line current pulses (see next section).

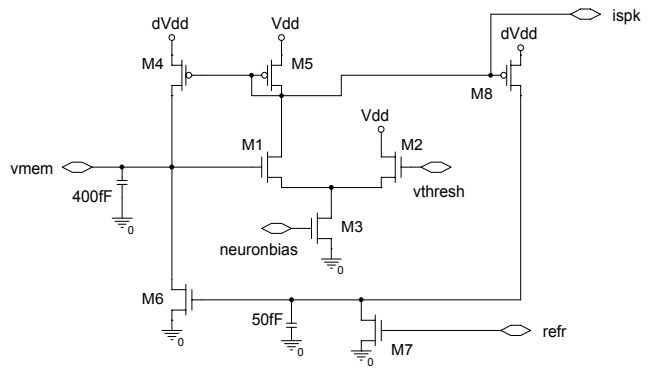

Fig. 5. Neuron circuit. In this circuit, input current is injected at the node vmem. Once vmem is charged close to vthresh, a positive feedback current produces an all-or-nothing event. The positive feedback current is mirrored out via ispk and the current is also used to charge the refractory timing circuit controlled by refr. (W/L values in microns: $\mathrm{M} 1, \mathrm{M} 2=2.4 / 1.8 ; \mathrm{M} 3=2.4 / 2.1 ; \mathrm{M} 4=3.9 / 2.4$; $\mathrm{M} 5=3.6 / 3.6 ; \mathrm{M} 6, \mathrm{M} 7=2.4 / 1.8 ; \mathrm{M} 8=3.0 / 2.4$ )

\section{Unarbitrated, Current-mode, Address-Event Output}

In addition to separating the analog and digital power supply lines, we further implemented a current-mode form of digital address readout. With each spike, the current pulse that is used to reset the neuron is amplified and mirrored to generate the appropriate bit pattern of current on the address lines. These current lines are held at $\mathrm{Vdd} / 2$ by an external circuit, preventing large scale voltage swings that could feedback into to the analog filtering stages.

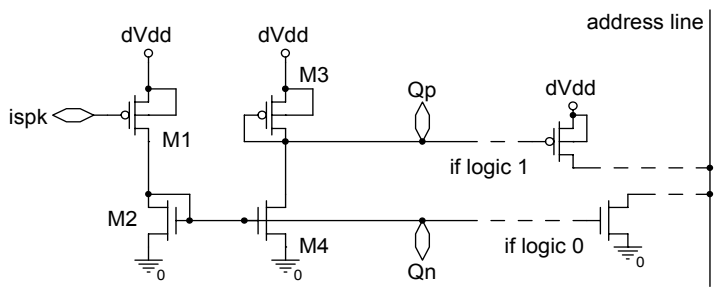

Fig. 6. Current-mode address lines. ispk is part of the neuron output current mirror and it is connected to a transistor that is considerably wider than the input transistor of the mirror to amplify the current. The current spike is used to inject or pull current from the address lines based on the particular neuron address. Where a ' 0 ' is desired, the Qn output is connected to an nFET to pull current from the address line and where a ' 1 ' is desired, the Qp output is connected to a pFET to inject current. $(\mathrm{W} / \mathrm{L}$ values are given in microns: $\mathrm{M} 1=12 / 1.5 ; \mathrm{M} 2=2.4 / 2.4 ; \mathrm{M} 3=$ $7 / / 2 \Delta \cdot M \Delta=2 n / 181$

\section{Testing Results}

The chip was fabricated using a commercially-available 0.5 $\mu \mathrm{m}$ 2-poly, 3-metal process. We conducted all testing at a supply voltage of $5 \mathrm{~V}$.

\section{A. Filters}

In Figures 7 and 8, we show the filter tuning curves for two parameter sets taken with a $1.4 \mathrm{mV}$ amplitude signal. Carefully avoiding oscillation, we were able to obtain $Q$ values as high as 65 on the most selective channel.

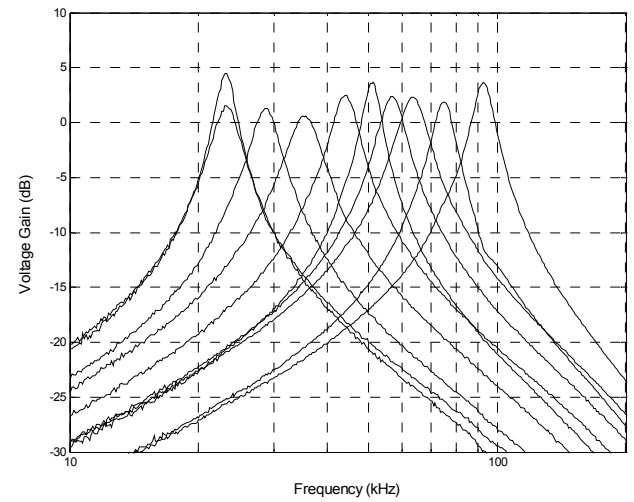

Fig. 7. Low-Q Filter tuning curves: The ten filters of one filterbank have their outputs directly accessible and tuning curves were measured. The quality factors in the array vary from 6.4 to 12.6 .

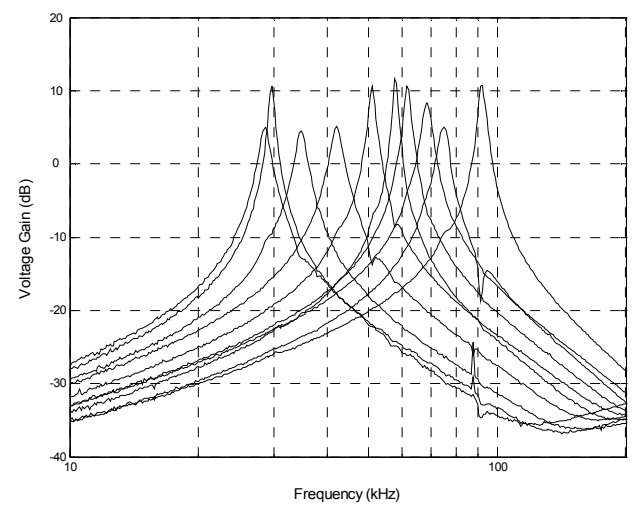

Fig. 8. High-Q Filter tuning curves: The filters were then tuned to be at a higher range of $\mathrm{Q}$ values. The quality factors at this setting vary from 18.6 to 48.7 .

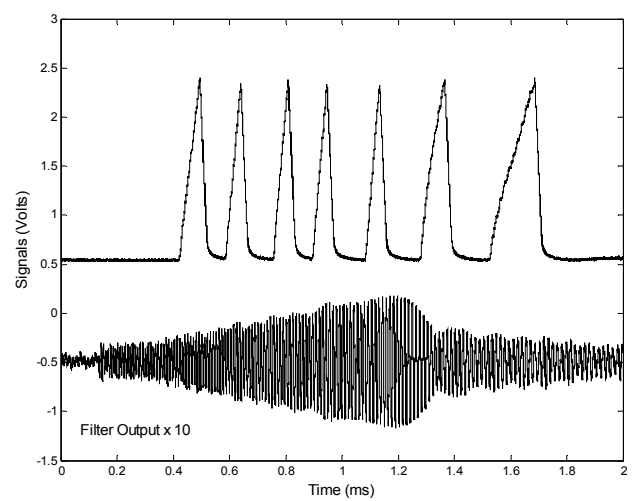

Fig. 9. Bottom: Filter response to an input frequency sweep that passes through the filter's center frequency. The temporal derivative of the filter's output is rectified and drives the neuron. Top: The neuron's membrane potential in response to the input frequency sweep. The membrane voltage is reset following each spike.

\section{B. Neurons}

We verified the neuron's functionality through observation of the neuron membrane voltage (vmem in Figure 5) to continuous and time-varying input. Figure 9 shows an example of the membrane potential response to a signal from the filter. 
A "threshold" current is discernible due to the rectifier circuit. In this chip, a single neuron is intended to represent the population of neurons in the biological cochlea that serves a particular region of the basilar membrane. As a result, the firing rate for the silicon neuron needs to be considerably higher than biological neurons. The refractory period was set to be minimal to obtain higher firing rates.

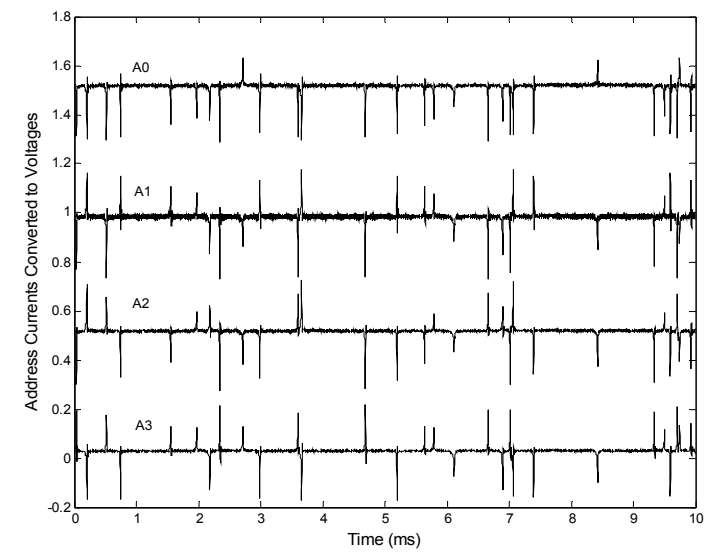

Fig. 10. Example address currents as a function of time. We convert the currents on the address lines to voltages using a current-sense amplifier. Because the individual encoder circuits do not need to charge the address lines up and down, the signaling has the potential to be fast. The upward voltage excursions represent ' 0 ' and downward excursions represent ' 1 '.

\section{Address-Event Output}

The current-mode output of the addresses is shown in Figure 10. With every neural spike, a current is injected or drawn from the four address lines. By reading the bit patterns, we extract the neuron address. Variations in bit-current amplitudes are due to transistor mismatch and are not address collisions. It should be noted that the two filterbanks have independent address outputs to reduce the potential for address collisions. Although we have not done so, it should be possible to design address codes where collisions were correctable.

Much of the design focused on avoiding feedback between the analog and digital systems so we inspected the filter outputs for evidence that the neuron spikes were generating interference in the filters, but found none.

\section{A Bat Echolocation Call}

Figure 11 shows the response of the filters and the spikes generated to a characteristic hyperbolic frequency sweep signal emitted by the big brown bat.

\section{DiscUSSION}

The ultrasonic filterbank chip demonstrated here provides moderate $\mathrm{Q}$ factors and generates output spike trains related to the amplitude of the signal, representing the population response of the auditory nerve.

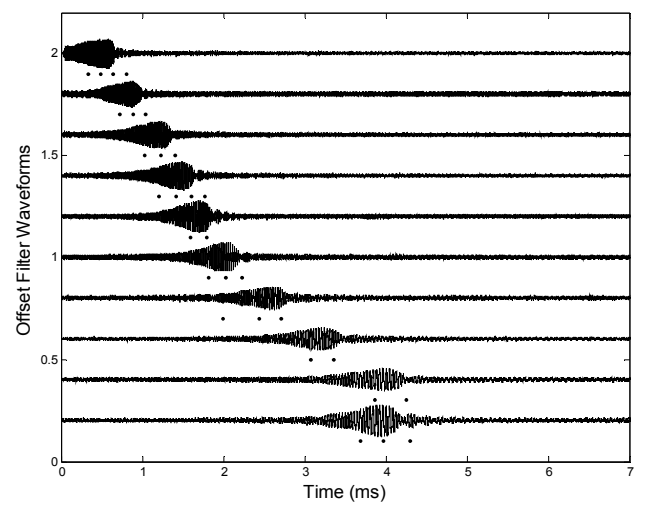

Fig. 11. Waveforms from the ten filters in response to a rapid downward hyperbolic frequency sweep from $100 \mathrm{kHz}$ to $4 \mathrm{kHz}$. Spikes produced by the neuron connected to each filter channel are shown just below each waveform. Notice that the neurons for higher frequencies fire a little more vigorously for similar amplitudes.

There are several improvements to be made, most notably in transistor matching. We also believe that a more sophisticated neuron should be used to produce sharper neural spikes at the output, separating the current levels used for the neuron and those used to report the address.

\section{ACKNOWLEDGMENT}

We thank Matt Cheely for some of the testing on the first version of this chip, laying the foundation for improvements.

\section{REFERENCES}

[1] A. N. Popper and R. R. Fay, "Hearing by Bats," in Springer Handbook of Auditory Research, vol. 5. New York: Springer-Verlag, 1995.

[2] R. F. Lyon and C. Mead, "An analog electronic cochlea," Acoustics, Speech, and Signal Processing [see also IEEE Transactions on Signal Processing], IEEE Transactions on, vol. 36, pp. 1119-1134, 1988.

[3] J. O. Pickles, An Introduction to the Physiology of Hearing, 2nd ed. San Diego: Academic Press, 1996.

[4] L. Watts, D. A. Kerns, R. F. Lyon, and C. A. Mead, "Improved implementation of the silicon cochlea," Solid-State Circuits, IEEE Journal of, vol. 27, pp. 692-700, 1992

[5] A. Van Schaik and E. Fragniere, "Pseudo-voltage domain implementation of a 2-dimensional silicon cochlea," presented at Circuits and Systems, 2001. ISCAS 2001. The 2001 IEEE International Symposium on, 2001.

[6] R. Sarpeshkar, R. F. Lyon, and C. A. Mead, "An analog VLSI cochlea with new transconductance amplifiers and nonlinear gain control," presented at Circuits and Systems, 1996. ISCAS '96., 'Connecting the World'., 1996 IEEE International Symposium on, 1996.

[7] R. Sarpeshkar, R. F. Lyon, and C. A. Mead, "Nonvolatile correction of Q-offsets and instabilities in cochlear filters," presented at Circuits and Systems, 1996. ISCAS '96., 'Connecting the World'., 1996 IEEE International Symposium on, 1996.

[8] P. M. Furth and A. G. Andreou, "Cochlear models implemented with linearized transconductors," presented at Circuits and Systems, 1996. ISCAS '96., 'Connecting the World'., 1996 IEEE International Symposium on, 1996.

[9] P. M. Furth, "A continuous-time bandpass filter implemented in subthreshold CMOS with large-signal stability," presented at Midwest Symposium on Circuits and Systems, Sacramento, CA, 1997.

[10] A. van Schaik, E. Fragniere, and E. Vittoz, "A silicon model amplitude modulation in the auditory brainstem," in Advances in Neural Info. Process. Syst., vol. 9, pp 741-747, Cambridge, MA, MIT Press, 1997. 